\title{
Probabilistic analysis of system outage in distributed antenna systems with composite channels
}

\author{
Jie Cai ${ }^{*},{ }$ Xibin $Z_{\text {hao }}{ }^{1}$, Jinyuan Wang ${ }^{2}$ and Ming Gu${ }^{1}$
}

\begin{abstract}
Distributed antenna systems (DAS) play a key role in future wireless communications. Despite the increasing previous research work, the channel models of DAS lack generality. This paper presents a holistic and generic channel model, which incorporates path loss, lognormal shadowing, and generalized-gamma fast fading into consideration. This overarching composite channel model encompasses well-known composite channel models as special cases. A probabilistic system outage model is attained by selective transmission scheme in terms of the proposed channel model and the distribution of mobile stations in the cell. Experimental results substantiate that the theoretical model provides sufficient precision for evaluating the outage performance of DAS.
\end{abstract}

Keywords: Distributed antenna system, Generic channel model, Outage probability analysis, Selective transmission scheme

\section{Introduction}

Due to the limited wireless resources, the transmission distance cannot be too large if the transmission rate is very large. To have a reasonable coverage area, antennas that are centrally attached to the base station in colocated antenna systems should be distributed throughout the cell so that the access distance for mobile stations (MSs) can be shortened. Such a system is called a distributed antenna system (DAS). Extensive studies have shown that DAS can enhance coverage, improve capacity, reduce outage, etc. [1-3]. Without any doubt, DAS will become a very promising candidate in future wireless communications.

Recently, the channel modeling and performance analysis of DAS have drawn significant attention. Assuming that the path loss is a constant, both the uplink and downlink capacities of DAS were investigated in [4]. This assumption is not always reasonable because the distances from the MS to each distributed antenna are usually different. Therefore, the path loss cannot be ignored. Zhu [5]

\footnotetext{
${ }^{*}$ Correspondence: caj08@mails.tsinghua.edu.cn

1 Key Laboratory for Information System Security of Ministry of Education,

School of Software, Tsinghua University, Beijing 100084, China

Full list of author information is available at the end of the article
}

analyzed the spectrum efficiency of DAS, but the shadowing was ignored. Due to the large space among distributed antennas, it is not reasonable to assume that all antennas experience the same shadowing. Nikolopoulos et al. [6] investigated the narrowband fading in indoor DASs, which only considered the fast fading. However, experimental measurements have shown that fast fading and shadowing occur simultaneously in practical systems [7]. Therefore, composite channel models should be considered for performance analysis. Furthermore, [8] investigated the channel model which combines the fast fading and lognormal shadowing effects. Then, [9-11] considered channel models which take path loss, lognormal shadowing, and Rayleigh fading into account. Chen et al. [12] and de Lima et al. [13] analyzed the system performance of DAS over composite shadowed-Nakagami-m fading channels. Although [9-13] considered composite channels, the fast fading was modeled by Rayleigh and Nakagami-m, which are special cases of the generalizedgamma distribution [14], and thus, the derived results lack generality.

In addition to the channel model, the distribution of MS is another important factor that should be considered. As is well known, the distribution of MS has strong

\section{是 Springer}

(c) 2013 Cai et al.; licensee Springer. This is an Open Access article distributed under the terms of the Creative Commons Attribution License (http://creativecommons.org/licenses/by/2.0), which permits unrestricted use, distribution, and reproduction in any medium, provided the original work is properly cited. 
impact on system performance. However, some previous works assumed that the position of MS is pre-given, so they did not consider the impact of the position of MS on system performance $[10,12]$. The other previous studies assumed that the MS is uniformly distributed in the cell [15-17]. However, for some scenarios, such as dense urban scenario, office building scenario, et al., the distribution of the MS may form a hot zone, and thus, the uniform distribution of MS may not always be a practical situation. Therefore, it is of interest and necessary to study the impact of the distribution of MS on system performance.

Motivated by the aforementioned literatures, we present a new composite channel model for DAS in this paper, which is represented as a mixture of path loss, lognormal shadowing, and generalized-gamma fast fading. The channel model in this paper is a generic model that covers many well-known composite channel models, such as Nakagami-lognormal, Rayleigh-lognormal, and Weibulllognormal. Based on this channel model, the probability density function (PDF) of the output signal-to-noise ratio (SNR) is derived. After that, we analyze the outage probability for the MS over a given position by selective transmission (ST) scheme. Furthermore, considering the distribution of MS in the system, an approximate theoretical expression of system outage probability is derived.

The remainder of this paper is organized as follows. System model is described in Section 2. In Section 3, an approximate expression of system outage probability is derived. Numerical results are presented in Section 4 before conclusions are drawn in Section 5.

\section{System model}

Consider a DAS with a single cell whose covering area is a circle with radius $R$, as shown in Figure 1 . The distributed antennas are arbitrarily distributed in the coverage of the cell, denoted as DA $i(i=1,2, \cdots, N)$, and are connected to a central unit via fiber optics. Only one antenna is employed at the MS. The positions of the MS and the DA $i$ are denoted by the polar coordinates $(\rho, \theta)$ and $\left(D_{i}, \theta_{i}\right)$, respectively. When the reference point is chosen to be the cell center, $\rho, D_{i}$ and $\theta, \theta_{i}$ are the distances and angles of the MS and the DA $i$ to the cell center.

Without the loss of generality, this paper focuses on the uplink transmission. Mathematically, for all DA $i(i=1$, $2, \cdots, N)$, the received uplink signals from the MS located at $(\rho, \theta)$ can be expressed as

$$
\begin{aligned}
\mathbf{Y} & =\left[y_{1}, y_{2}, \cdots, y_{N}\right]^{T} \\
& =\sqrt{E}\left[h_{1}, h_{2}, \cdots, h_{N}\right]^{T} x+\mathbf{Z} \in \mathbb{C}^{N \times 1},
\end{aligned}
$$

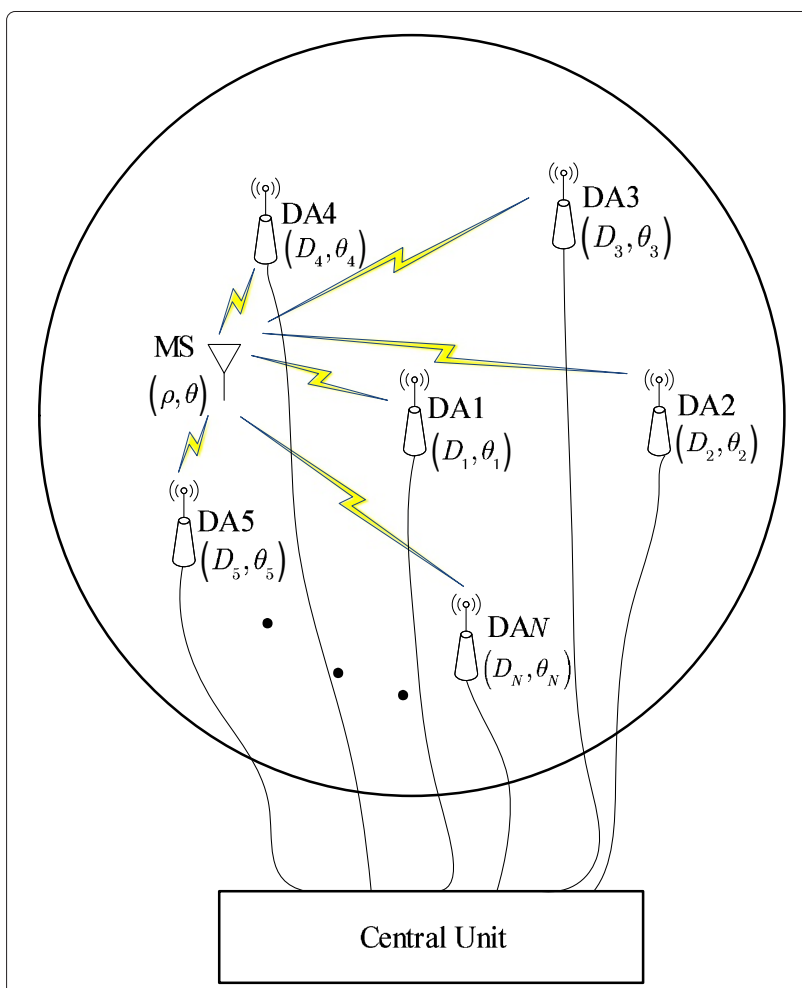

Figure 1 Structure of a distributed antenna system.

where the $i$ th element of $\mathbf{Y}, y_{i}$, denotes the received signal at the DA $i, E$ is the transmit signal power of the MS, $h_{i}$ represents the channel fading between the MS and the DA $i, x$ is the transmit symbol of the MS with unit energy, $\mathbf{Z}$ is the complex white Gaussian noise vector with zero mean and variance $N_{0}$.

Generally, the evaluation for channel fading $h_{i}$ is discussed in [18]. In this paper, we follow their idea and evaluate $h_{i}$ by the following formula:

$$
h_{i}=G_{i} \sqrt{L_{i}(\rho, \theta) \cdot \Omega_{i}}, \forall i \in\{1,2, \cdots, N\}
$$

where $G_{i}$ denotes the fast fading between the DA $i$ and the MS. Assuming that the envelope of $G_{i}$ undergoes an independent but not identical generalized-gamma distribution [14]

$$
f_{\left|G_{i}\right|}(g)=\frac{2 v_{i} m_{i}^{m_{i}} g^{2 m_{i} v_{i}-1}}{\Gamma\left(m_{i}\right)} \exp \left(-m_{i} g^{2 v_{i}}\right), g \geq 0
$$

where $m_{i} \geq 1 / 2$ is the fast fading parameter, $v_{i}>0$ is the shape parameter, and $\Gamma(x)=\int_{0}^{\infty} t^{x-1} e^{-t} \mathrm{~d} t$ is the gamma function. Note that the generalized-gamma distribution covers several commonly used fading distributions as special cases, which can be seen in Table 1 . Moreover, $L_{i}(\rho, \theta)$ 
Table 1 Some special cases of generalized-gamma distribution

\begin{tabular}{ccc}
\hline Envelope distribution & $\boldsymbol{m}_{\boldsymbol{i}}$ & $\boldsymbol{v}_{\boldsymbol{i}}$ \\
\hline Rayleigh & 1 & 1 \\
\hline Nakagami-m & 1 & 1 \\
\hline Weibull & & $1 / 2$ \\
\hline Gamma & 1 & $1 / 2$ \\
\hline Exponential & $1 / 2$ & 1 \\
\hline Half-normal & $m_{i} \rightarrow \infty$ & 1 \\
\hline AWGN & $m_{i} \rightarrow \infty$ & $v_{i} \rightarrow 0$ \\
\hline Lognormal & &
\end{tabular}

in Equation (2) denotes the path loss between DA $i$ and $(\rho, \theta)$, and it can be derived by [19]

$$
L_{i}(\rho, \theta)=\left(\frac{d_{0}}{d_{i}(\rho, \theta)}\right)^{\beta_{i}},
$$

where $d_{0}$ is the reference distance, $\beta_{i}$ is the path loss exponent, $d_{i}(\rho, \theta)$ denotes the distance between DA $i$ and the MS, which can be expressed as $d_{i}(\rho, \theta)=$ $\sqrt{\rho^{2}+D_{i}^{2}-2 \rho D_{i} \cos \left(\theta-\theta_{i}\right)}$.

In addition, $\Omega_{i}$ in Equation (2) represents the shadow fading, and it can be modeled by a lognormal distribution [7]

$$
f_{\Omega_{i}}(\omega)=\frac{\xi}{\sqrt{2 \pi} \sigma_{i} \omega} \exp \left[-\frac{\left(10 \log _{10} \omega-\mu_{i}\right)^{2}}{2 \sigma_{i}^{2}}\right], \omega>0,
$$

where $\xi=10 / \ln 10, \mu_{i}($ in $\mathrm{dB})$ and $\sigma_{i}($ in $\mathrm{dB})$ are the mean and standard deviation of $10 \log _{10} \omega$.

From Equations (1) and (2), the output SNR at DA $i$ can be obtained as

$$
\gamma_{i}=\frac{E\left|h_{i}\right|^{2}}{N_{0}}=\frac{E L_{i}(\rho, \theta) \Omega_{i}\left|G_{i}\right|^{2}}{N_{0}}
$$

Based on the definition for $\gamma_{i}$, its PDF could be computed. The following lemma gives the analytic form of $f_{\gamma_{i}}$.

Lemma 2.1. Let $S_{i} \triangleq L_{i}(\rho, \theta) \Omega_{i} / N_{0}$ in Equation (7), the PDF of $\gamma_{i}$ can be expressed as where $\tilde{\mu}_{i}(\rho, \theta)=\mu_{i}+10 \log _{10}\left[E L_{i}(\rho, \theta) / N_{0}\right]$.

The proof of Lemma 2.1 can be found in the 'Appendix' section.

\section{System outage probability analysis}

Assuming that the ST scheme is applied, i.e., only one link is selected for transmission to maximize the output SNR

$$
\gamma=\max \left\{\gamma_{1}, \gamma_{2}, \cdots, \gamma_{N}\right\} .
$$

Owing to the large space among distributed antennas, it is reasonable to assume all the links between distributed antennas and the MS undergo independent shadowing and fast fading, i.e., $\gamma_{i}$ in Equation (8) is independent of $\gamma_{j}(i \neq j)$. Accordingly, the outage probability for the MS located at $(\rho, \theta)$ can be obtained as

$$
\begin{aligned}
\delta(\rho, \theta) & =\operatorname{Pr}\left(\gamma<\gamma_{\text {th }}\right) \\
& =\operatorname{Pr}\left(\gamma_{1}<\gamma_{\text {th }}, \cdots, \gamma_{N}<\gamma_{\text {th }}\right) \\
& =\prod_{i=1}^{N} \operatorname{Pr}\left(\gamma_{i}<\gamma_{\text {th }}\right),
\end{aligned}
$$

where $\gamma_{\text {th }}$ is the threshold of SNR. Basically, the formula above states that the value of $\delta(\rho, \theta)$ is determined by the probability of each $\operatorname{Pr}\left(\gamma_{i}<\gamma_{\text {th }}\right)$ and the latter one could be estimated as in the following theorem.

Theorem 3.1. The outage probability between the MS and the DAi $\operatorname{Pr}\left(\gamma_{i}<\gamma_{\text {th }}\right)$ can be expressed as

$$
\operatorname{Pr}\left(\gamma_{i}<\gamma_{\text {th }}\right) \cong 1-\frac{\sum_{n=1}^{N_{p}} H_{n} \Gamma\left(m_{i}, m_{i}\left(\frac{\gamma_{\text {th }}}{10 \frac{\sqrt{2} \sigma_{i} t_{n}+\tilde{\mu}_{i}(\rho, \theta)}{10}}\right)^{\nu_{i}}\right)}{\sqrt{\pi} \Gamma\left(m_{i}\right)},
$$

where $t_{n}$ and $H_{n}$ are the base points and weight factors of the $N_{p}$-order Hermite polynomial, respectively.

The proof of Theorem 3.1 can be found in the 'Appendix' section.

Furthermore, by substituting Equation (10) into Equation (9), the outage probability in Equation (9) can be approximated as

$$
\delta(\rho, \theta) \cong \prod_{i=1}^{N}\left[1-\frac{\sum_{n=1}^{N_{p}} H_{n} \Gamma\left(m_{i}, m_{i}\left(\frac{\gamma_{\mathrm{th}}}{10 \frac{\sqrt{2} \sigma_{i} t_{n}+\tilde{\mu}_{i}(\rho, \theta)}{10}}\right)^{v_{i}}\right)}{\sqrt{\pi} \Gamma\left(m_{i}\right)}\right] .
$$

$$
\begin{aligned}
f_{\gamma_{i}}(r) & =\int_{0}^{\infty} f_{\gamma_{i} \mid S_{i}}(r \mid s) f_{S_{i}}(s) \mathrm{d} s \\
& =\int_{0}^{\infty} \frac{v_{i} m_{i} m_{i} r^{m_{i} v_{i}-1}}{s^{m_{i} v_{i}} \Gamma\left(m_{i}\right)} \exp \left[-m_{i}\left(\frac{r}{s}\right)^{v_{i}}\right] \cdot \frac{\xi}{\sqrt{2 \pi} \sigma_{i} s} \exp \left[-\frac{\left(10 \log _{10} s-\tilde{\mu}_{i}(\rho, \theta)\right)^{2}}{2 \sigma_{i}^{2}}\right] \mathrm{d} s,
\end{aligned}
$$


Note that the outage probability in Equation (11) is a function of the position of MS. Theoretically, the system outage probability also relates to the distribution of the MS in the cell. Assuming that $r(\rho, \theta)$ (in polar coordinates) is the PDF that used to describe the distribution of the MS in the cell, the system outage probability can be expressed as

$$
P_{\text {out }}=\mathrm{E}_{\rho, \theta}[\delta(\rho, \theta)]=\int_{0}^{2 \pi} \int_{0}^{R} \delta(\rho, \theta) r(\rho, \theta) \rho \mathrm{d} \rho \mathrm{d} \theta .
$$

Since the distribution of the MS is arbitrary, the expression in Equation (12) is complex and usually has no closed-form solution. By employing the composite Simpson's rule [20], the final uplink system outage probability can be derived as

$$
P_{\text {out }} \cong \frac{h k}{9} \sum_{p=0}^{P} \sum_{q=0}^{Q}\left[c_{p, q} \rho_{p} r\left(\rho_{p}, \theta_{q}\right) \delta\left(\rho_{p}, \theta_{q}\right)\right],
$$

where the two even number $P$ and $Q$ are chosen to determine the step sizes $h=R / P$ and $k=2 \pi / Q$, respectively. In addition, $\rho_{p}=p h,(p=0,1,2, \cdots, P)$ and $\theta_{q}=$ $q k,(q=0,1,2, \cdots, Q)$. The weigh factor $c_{p, q}$ is the element of matrix $\mathbf{C}$, in the $(p+1)^{\text {th }}$ row and $(q+1)^{\text {th }}$ column. The matrix $\mathbf{C}$ can be found in [20].

\section{Numerical results}

In this section, we will verify the accuracy of the approximate expression of system outage probability by Monte Carlo simulations. Without the loss of generality, a singlecell DAS with a radius of $R$ is used as the test system. In the system, one antenna is located in the center of the cell, and other antennas are evenly and symmetrically placed on a circle with a radius of $2 R / 3$. For the sake of simplicity, some parameters are assumed to be the same, i.e., $\beta=$ $\beta_{i}, \sigma=\sigma_{i}, m=m_{i}, \mu=\mu_{i}, v=v_{i}$ for $i=1,2, \cdots, N$.

Furthermore, to describe the non-uniformity of the MS in the cell, we divide the whole cell into two regions, as shown in Figure 2. Region 1 (denoted as $\Psi_{1}$ ) is the circular area, which is in the center of the cell and with a radius of $R_{\mathrm{h}}$, and the residual annular zone is region 2 (denoted as $\Psi_{2}$ ). Therefore, the PDF for describing the distribution of the MS in the cell can be supposed as

$$
r(\rho, \theta)=\left\{\begin{array}{cl}
\frac{\lambda}{S_{\mathrm{h}}} & (\rho, \theta) \in \Psi_{1} \\
\frac{1-\lambda}{S-S_{\mathrm{h}}} & (\rho, \theta) \in \Psi_{2}
\end{array},\right.
$$

where $S_{\mathrm{h}}=\pi R_{\mathrm{h}}^{2}$ is the area of region 1 while $S=\pi R^{2}$ is the area of the whole cell. $\lambda \in[0,1]$ is the probability that the MS distributed in region 1 . It can be observed that, when $\lambda=S_{\mathrm{h}} / S$, the MS in the cell follows uniform distribution; when $\lambda>S_{\mathrm{h}} / S$, region 1 is the hot zone, the probability of the MS which located in region 1 increases;

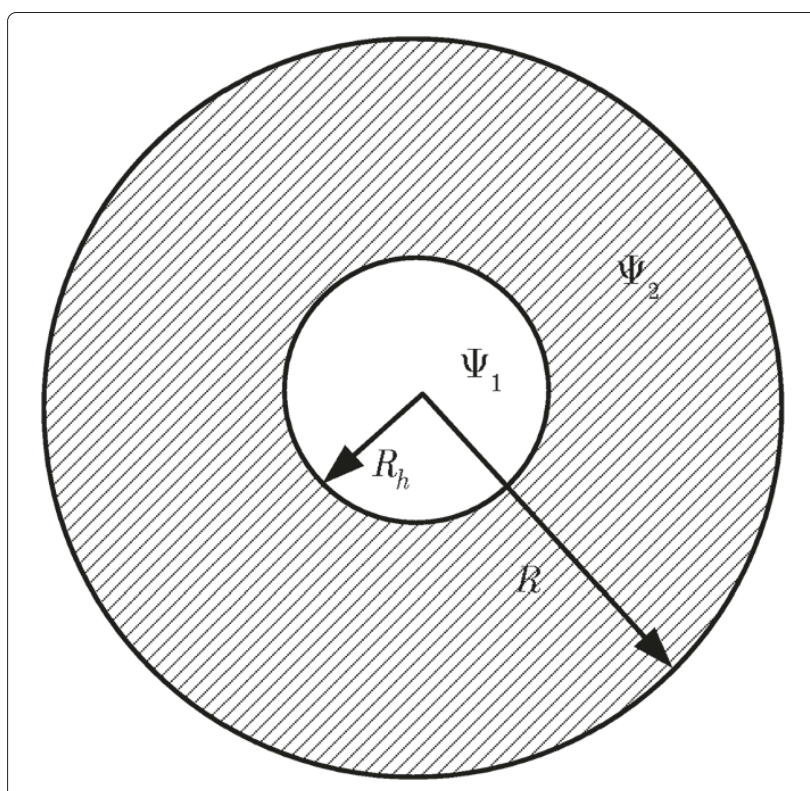

Figure 2 The distribution of MSs in the cell.

when $\lambda<S_{\mathrm{h}} / S$, the probability of the MS which located in region 2 increases. In addition, the main simulation parameters are listed in Table 2.

Figures 3, 4, 5, 6 and 7 show the system outage probability versus the transmit $\operatorname{SNR}\left(E / N_{0}\right)$ in different scenarios. Obviously, with the increase of the transmit SNR, the received SNR will increase, and thus, the system outage probability will decrease. Specifically, Figure 3 shows the system outage probability versus transmit SNR $\left(E / N_{0}\right)$ with different numbers of distributed antennas $(N)$. Obviously, the system outage probability drops with the increase of $N$. When the value of $N$ is larger, a higher

Table 2 Main simulation parameters

\begin{tabular}{ccc}
\hline Parameters & Symbol & Value \\
\hline Radius of the cell & $R$ & $500 \mathrm{~m}$ \\
\hline Radius of region 1 & $R_{\mathrm{h}}$ & $125 \mathrm{~m}$ \\
\hline Reference distance & $d_{0}$ & $20 \mathrm{~m}$ \\
\hline Path loss exponent & $\beta$ & 4 \\
\hline Number of distributed antennas & $N$ & 7 \\
\hline Mean of shadow fading & $\mu$ & $0 \mathrm{~dB}$ \\
\hline Standard deviation of shadow fading & $\sigma$ & $8 \mathrm{~dB}$ \\
\hline Fast fading parameter & $m$ & 1 \\
\hline Shape parameter & $v$ & 2 \\
\hline Probability that MSs distributed in region 1 & $\lambda$ & 0.0625 \\
\hline Order of Hermite polynomial & $N_{p}$ & 40 \\
\hline Number of equidistant nodes for polar radius & $P$ & 10 \\
\hline Number of equidistant nodes for polar angle & $Q$ & 10 \\
\hline
\end{tabular}




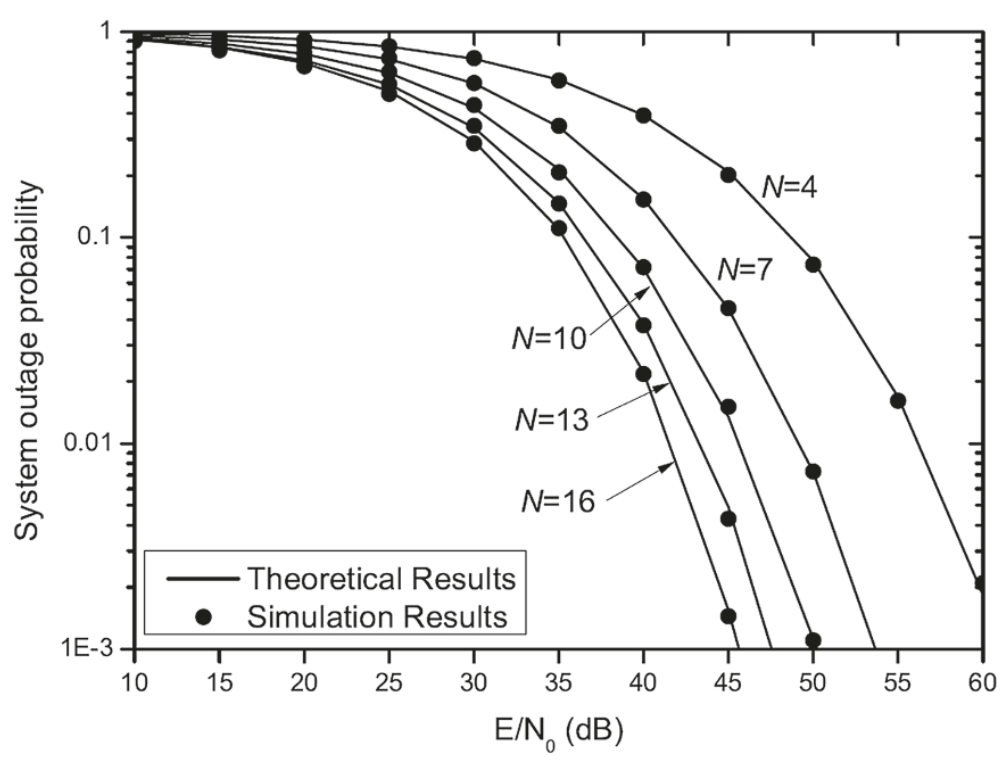

Figure 3 System outage probability versus transmit SNR with different $N$.

diversity gain can be achieved, which will result in a higher output SNR. Therefore, a lower outage probability can be achieved. Furthermore, the gaps between every two neighboring $N$ become narrow with the increase of $N$. The improvement of outage performance becomes very slight when $N$ increases to a certain extent. Therefore, too many distributed antennas in one cell are not necessary.

Figure 4 depicts the system outage probability as a function of the radius of the cell $(R)$. This figure indicates that the system outage performance can be improved with the decrease of $R$. Just as is expected, the smaller the coverage of the cell is, the better the system outage performance becomes. Moreover, the performance gaps between every two neighboring $R$ enlarge with the decrease of $R$. Generally, we hope the coverage of the cell can be as large as possible, but a large coverage of the cell will reduce the outage performance. Therefore, a tradeoff between coverage and outage performance should be considered in practical communication systems.

Figure 5 plots the system outage probability with different shape parameters $(v)$. In Figure 5 , it is depicted that the system outage performance improves with the increase

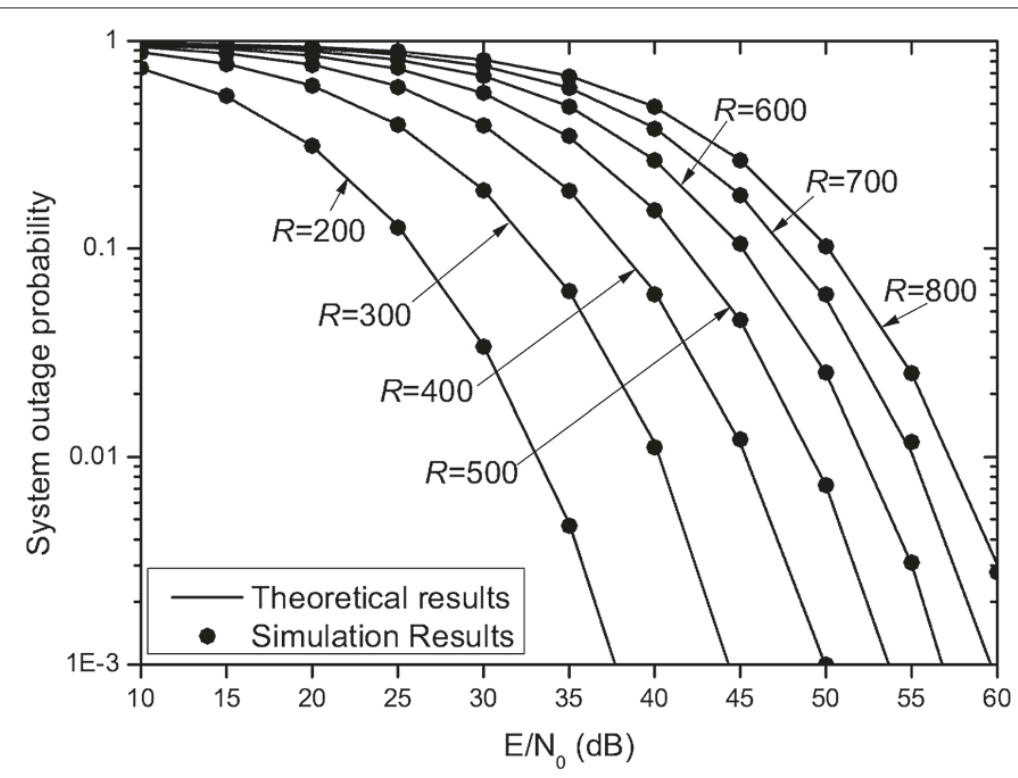

Figure 4 System outage probability versus transmit SNR with different $R$ (in $m$ ). 


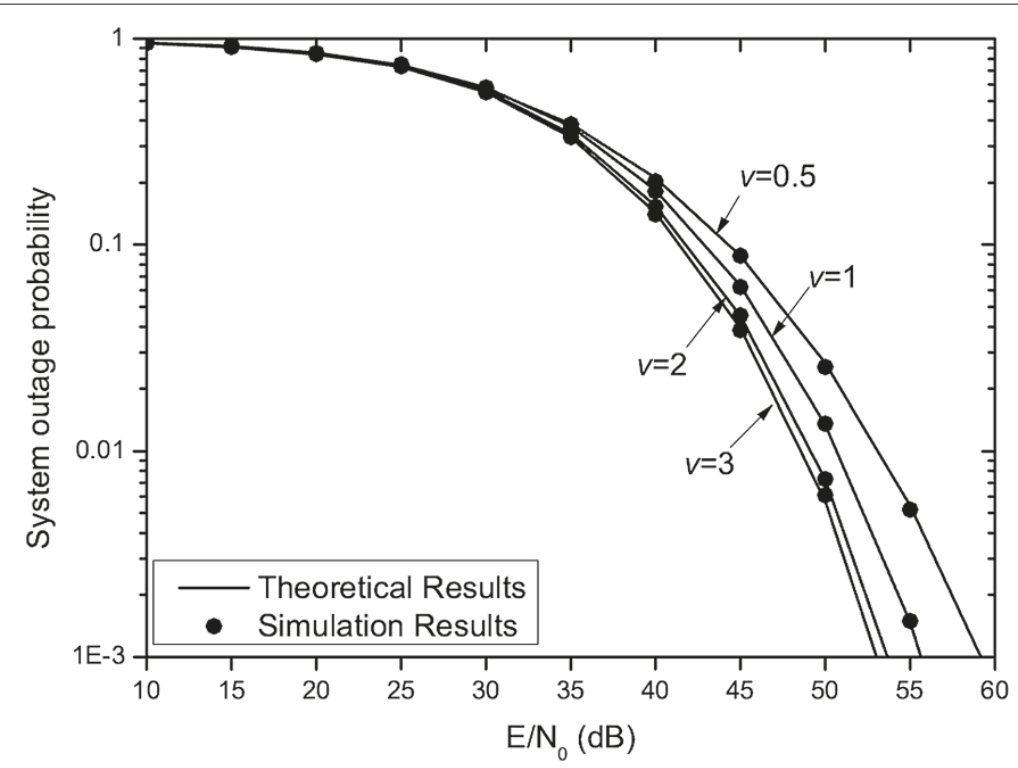

Figure 5 System outage probability versus transmit SNR with different $v$.

of the shape parameter $v$. Moreover, the gaps between every two neighboring $v$ become narrow with the increase of $v$. The improvement of outage performance becomes very slight when the shape parameter increases to a certain extent. Therefore, too large shape parameters are not necessary.

Figure 6 illustrates the relationship between the system outage probability and path loss exponent $(\beta)$. It can be seen that the system outage performance can be improved by decreasing the value of $\beta$. From Equation (4), it can be seen that the path loss increase with the decrease of $\beta$, and then, the channel gain will increase correspondingly. Therefore, the output SNR will become better. That is, the number of communication links which cannot satisfy the SNR threshold will be decreased. In other words, the outage performance is enhanced.

Figure 7 shows the impact of the distribution of the MS in the cell on system outage performance. Note that, when $\lambda=S_{\mathrm{h}} / S=0.0625$, the MS are uniformly distributed in the cell. When $\lambda>0.0625$, the probability of the MS which located in region 1 is higher than in region 2 . From Figure 7 , it can be observed that, with the increase of

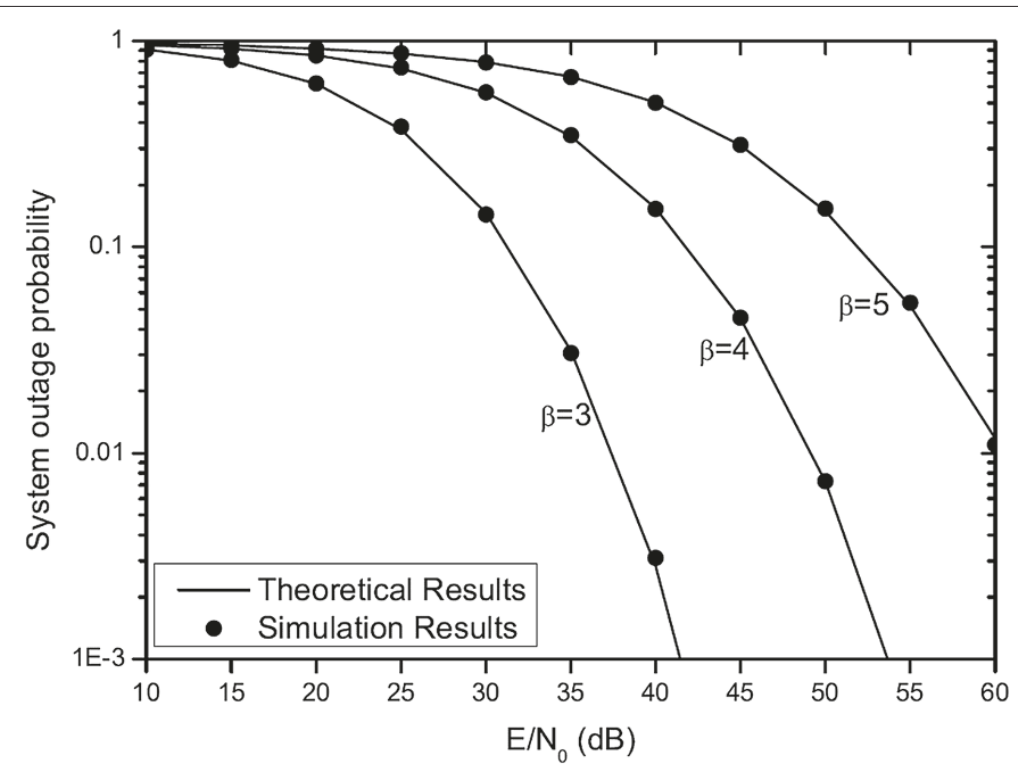

Figure 6 System outage probability versus transmit SNR with different $\beta$. 


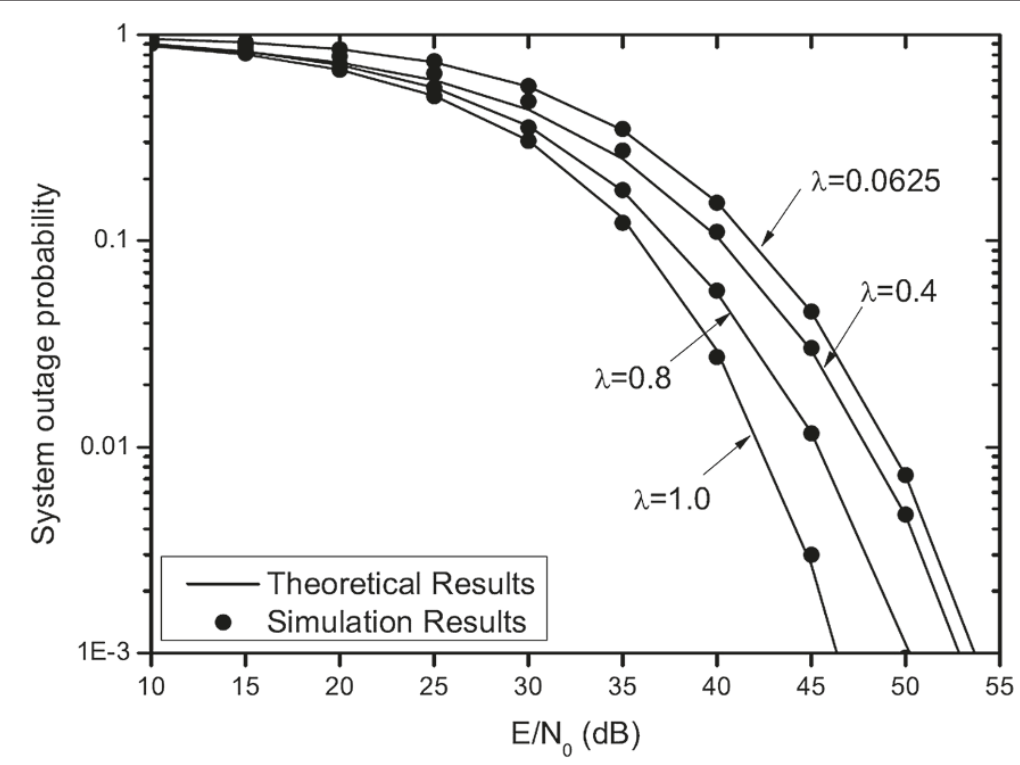

Figure 7 System outage probability versus transmit SNR with different $\lambda$.

the value $\lambda$ from 0.0625 to 1.0 , the probability of the MS which distributed in region 1 will increase. Since one distributed antenna is located in the center of the cell, the average access distance will reduce, and this will result in the decrease of system outage probability. It should be noted that the system outage probability varies with the value of $\lambda$, which indicates that the distribution of the MS has a strong impact on the system outage performance.

As is well known, the deployment of distributed antennas has strong impacts on system performance. In this simulation, two schemes, i.e., circular layout with central antenna scheme and circular layout without central antenna scheme, are proposed to show the optimal radius for antenna deployment. For circular layout with central antenna scheme, one antenna is deployed in the cell center and the other antennas are evenly deployed on a circle, while all antennas are evenly deployed on a circle for another scheme. Figure 8 shows system outage probability versus normalized radius for antenna deployment when MS is uniformly distributed. It can be seen that, when the normalized radius is less than 0.5 , the outage probability of the circular layout without central antenna scheme is smaller than that of the circular layout with central antenna scheme. However, when the normalized radius exceeds 0.5 , the circular layout with central antenna scheme outperforms the circular layout without central antenna scheme. From Figure 8, it can be observed that when the value of the normalized radius is about $2 / 3$, the minimum system outage probability can be derived for both the two schemes. In other words, the optimal radius for antenna placement is about $2 R / 3$ in this simulation scenario.
It should be noted from Figures 3, 4, 5, 6, 7 and 8 that the theoretical results and simulation results of the system outage probability are quite accordant with each other. Therefore, the approximate expression of the system outage probability shown in this paper can provide perfect approximation to the realistic outage performance of DAS without time-intensive simulations. Furthermore, this approximate expression can be used to evaluate the system outage probability in different scenarios, and it will lay a very good foundation for further research such as antenna placement and network planning.

\section{Conclusions}

In this paper, we presented a new channel model for DAS, called shadowed generalized-gamma composite channel. The channel fading in this model is a generic case of many classic models, such as Rayleigh-lognormal fading, Nakagami-lognormal fading, Weibull-lognormal, et al. Under such a channel model, we investigated the probabilistic characterization of uplink system outage with the ST scheme and the distribution of MS in the cell. Using the principle of numerical analysis, we derived an approximate theoretical expression of system outage probability. It has been validated by the numerical results, thus showing that the derived approximate expression can be applied to evaluate the outage probability of DAS precisely.

\subsection{Nomenclature}

$\begin{array}{ll}y_{i} & \text { received signal at DA } i \\ E & \text { transmit signal power of MS } \\ h_{i} & \text { channel fading between DA } i \text { and MS }\end{array}$




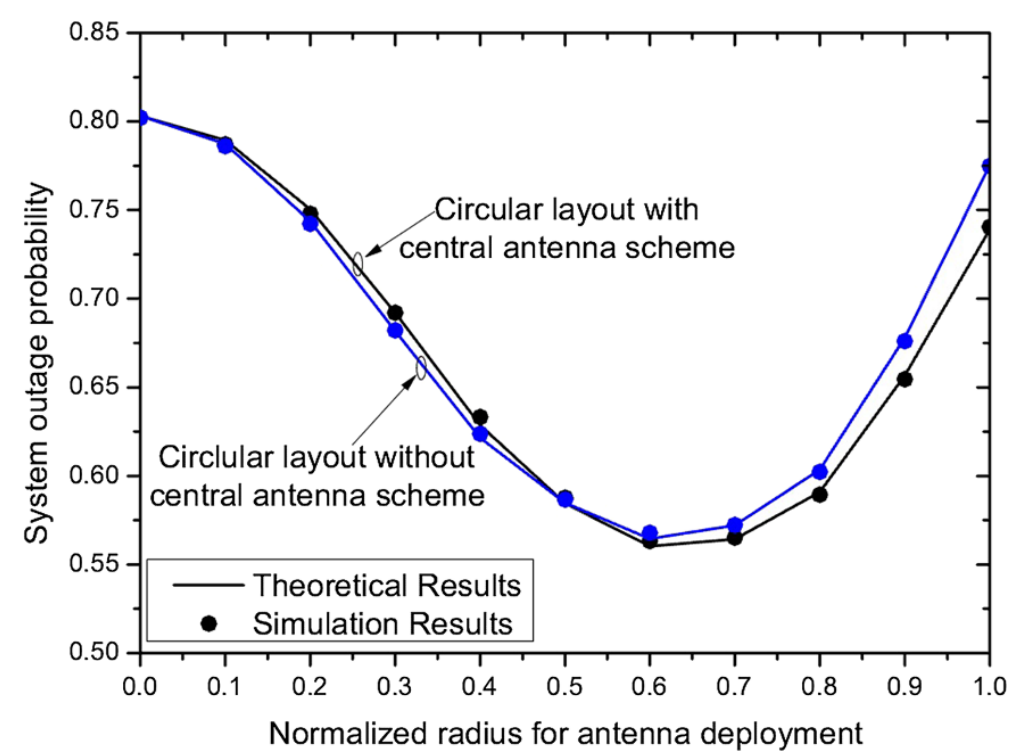

Figure 8 System outage probability versus normalized radius for antenna deployment when $E / N_{0}=30 \mathrm{~dB}, \lambda=0.0625$, and $N=7$.

$X \quad$ transmit symbol of MS with unit energy

$\mathbf{Z} \quad$ complex white Gaussian noise

$G_{i} \quad$ fast fading between DA $i$ and the MS

$v_{i} \quad$ shape parameter

$m_{i} \quad$ fast fading parameter

$\Gamma(x) \quad$ Gamma function

$L_{i}(\rho, \theta)$ path loss between DA $i$ and MS

$d_{0} \quad$ reference distance

$\beta_{i} \quad$ path loss exponent

$d_{i}(\rho, \theta)$ distance between DA $i$ and MS

$\Omega_{i} \quad$ shadow fading

$\xi \quad 10 / \ln 10$

$\mu_{i} \quad$ mean value(in $\mathrm{dB}$ ) of $10 \log _{10} \omega$

$\sigma_{i} \quad$ standard deviation of $10 \log _{10} \omega$

$N_{0} \quad$ the variance of white Gaussian noise

$\gamma_{i} \quad$ output SNA at DAi

$S_{i} \quad E L_{i}(\rho, \theta) \Omega_{i} / N_{0}$

$\tilde{\mu}_{i}(\rho, \theta) \quad \mu_{i}+10 \log _{10}\left[E L_{i}(\rho, \theta) / N_{0}\right]$

$\gamma \quad$ maximum output SNR

$\gamma_{\text {th }} \quad$ threshold of SNR

$\delta(\rho, \theta) \quad$ outage probability of MS

$t_{n} \quad$ base points of the $N_{p}$-order Hermite

polynomial

$H_{n} \quad$ weight factors of the $N_{p}$-order Hermite polynomial

$r(\rho, \theta) \quad$ PDF of MS

$\Gamma(z, t)$ incomplete Gamma function

$H_{n} \quad$ Hermite polynomial

$P_{\text {out }} \quad$ system outage probability

h $R / P$

$k \quad 2 \pi / Q$

\section{Appendix}

\section{Proof for Lemma 2.1}

Proof 1. Since, $\left|G_{i}\right|$ follows a generalized-famma distribution, the cumulative distribution function (CDF) of $\left|G_{i}\right|^{2}$ can be expressed as

$$
F_{\left|G_{i}\right|^{2}}(g)=\operatorname{Pr}\left(\left|G_{i}\right|^{2} \leq g\right)=\int_{-\sqrt{g}}^{\sqrt{g}} f_{\left|G_{i}\right|}(t) \mathrm{d} t .
$$

Differentiating Equation (15) with respect to $g$ yield the PDF of $\left|G_{i}\right|^{2}$ as

$$
\begin{aligned}
f_{\left|G_{i}\right|^{2}}(g) & =\frac{\mathrm{d} \int_{-\sqrt{g}}^{\sqrt{g}} f_{\left|G_{i}\right|}(t) \mathrm{d} t}{\mathrm{~d} g} \\
& =\frac{v_{i} m_{i}^{m_{i}} g^{m_{i} v_{i}-1}}{\Gamma\left(m_{i}\right)} \exp \left(-m_{i} g^{v_{i}}\right), g \geq 0 .
\end{aligned}
$$

Furthermore, $S_{i} \triangleq E L_{i}(\rho, \theta) \Omega_{i} / N_{0}$ in Equation (6), we can obtain that $\gamma_{i}=S_{i}\left|G_{i}\right|^{2}$. Determined by $\Omega_{i}$, $S_{i}$ also follows a lognormal distribution and its PDF can be derived as

$$
f_{S_{i}}(s)=\frac{\xi}{\sqrt{2 \pi} \sigma_{i} s} \exp \left[-\frac{\left(10 \log _{10} s-\tilde{\mu}_{i}(\rho, \theta)\right)^{2}}{2 \sigma_{i}^{2}}\right], s>0,
$$

where $\tilde{\mu}_{i}(\rho, \theta)=\mu_{i}+10 \log _{10}\left[E L_{i}(\rho, \theta) / N_{0}\right]$.

In the presence of $S_{i}$, the conditional CDF $F_{\gamma_{i} \mid S_{i}}(\gamma \leq r \mid s)$ can be written as

$$
F_{\gamma_{i} \mid S_{i}}(\gamma \leq r \mid s)=F_{\left|G_{i}\right|^{2}}\left(G \leq \frac{r}{s}\right)=\int_{0}^{\frac{r}{s}} f_{\left|G_{i}\right|^{2}}(g) \mathrm{d} g .
$$


Differentiating Equation (18) with respect to $r$ yield the conditional PDF of $f_{\gamma_{i} \mid S_{i}}(r \mid s)$ as

$$
f_{\gamma_{i} \mid S_{i}}(r \mid s)=\frac{1}{s} f_{\left|G_{i}\right|^{2}}\left(\begin{array}{l}
r \\
s
\end{array}\right)=\frac{v_{i} m_{i} m_{i} r^{m_{i} v_{i}-1}}{s^{m_{i} v_{i}} \Gamma\left(m_{i}\right)} \exp \left(-m_{i}\left(\frac{r}{s}\right)^{v_{i}}\right)
$$

By applying Equations (17) and (19), the PDF of $\gamma_{i}$ can be expressed as

$$
\begin{aligned}
f_{\gamma_{i}}(r)= & \int_{0}^{\infty} f_{\gamma_{i} \mid S_{i}}(r \mid s) f_{S_{i}}(s) \mathrm{d} s \\
= & \int_{0}^{\infty} \frac{v_{i} m_{i} m_{i} r^{m_{i} v_{i}-1}}{s^{m_{i} v_{i}} \Gamma\left(m_{i}\right)} \exp \left[-m_{i}\left(\frac{r}{s}\right)^{v_{i}}\right] \\
& \cdot \frac{\xi}{\sqrt{2 \pi} \sigma_{i} s} \exp \left[-\frac{\left(10 \log _{10} s-\tilde{\mu}_{i}(\rho, \theta)\right)^{2}}{2 \sigma_{i}^{2}}\right] \mathrm{d} s .
\end{aligned}
$$

\section{Proof for Theorem 3.1}

Before getting into the details of derivation, we mention two important propositions that are already known.

\section{Proposition 1.}

$$
\begin{aligned}
\int x^{m} e^{-\beta x^{n}} \mathrm{~d} x= & -\frac{\Gamma\left(\gamma, \beta x^{n}\right)}{n \beta^{\gamma}} \\
= & -\frac{1}{n \beta^{\gamma}} \int_{\beta x^{n}}^{\infty} t^{\gamma-1} e^{-t} \mathrm{~d} t, \\
& {\left[\gamma=\frac{m+1}{n}, \beta \neq 0, n \neq 0\right] }
\end{aligned}
$$

which can be found in Section 2.33 in [21].

\section{Proposition 2.}

$$
\int_{-\infty}^{\infty} e^{-x^{2}} f(x) \mathrm{d} x \cong \sum_{i=1}^{N_{p}} H_{n} f\left(t_{n}\right)
$$

which can be found in Equations $(25,4,46)$ in [22].

By Lemma 2.1, $\operatorname{Pr}\left(\gamma_{i}<\gamma_{\text {th }}\right)$ can be expressed as

$$
\begin{aligned}
\operatorname{Pr}\left(\gamma_{i}<\gamma_{\text {th }}\right) & =1-\int_{\gamma_{\text {th }}}^{\infty} f_{\gamma_{i}}(r) \mathrm{d} r \\
& =1-\int_{0}^{\infty} \frac{\xi}{\sqrt{2 \pi} \sigma_{i} s} \exp \left[-\frac{\left(10 \log _{10} s-\tilde{\mu}_{i}(\rho, \theta)\right)^{2}}{2 \sigma_{i}^{2}}\right] \\
& =\frac{v_{i} m_{i}{ }^{m_{i}}}{s^{m_{i} v_{i}} \Gamma\left(m_{i}\right)} \int_{\gamma_{\text {th }}}^{\infty} r^{m_{i} \nu_{i}-1} \exp \left[-m_{i}\left(\frac{r}{s}\right)^{v_{i}}\right] \mathrm{d} r \mathrm{~d} s .
\end{aligned}
$$

Therefore, by using proposition 1, Equation (22) can be further written as

$$
\begin{aligned}
\operatorname{Pr}\left(\gamma_{i}<\gamma_{\text {th }}\right)= & 1-\int_{0}^{\infty} \frac{\xi}{\sqrt{2 \pi} \sigma_{i} s} \exp \left[-\frac{\left(10 \log _{10} s-\tilde{\mu}_{i}(\rho, \theta)\right)^{2}}{2 \sigma_{i}^{2}}\right] \\
& \times \frac{v_{i} m_{i} m_{i}}{s^{m_{i} v_{i}} \Gamma\left(m_{i}\right)} \frac{\Gamma\left(m_{i}, m_{i}\left(\frac{\gamma_{\mathrm{th}}}{s}\right)^{v_{i}}\right)}{v_{i}\left(\frac{m_{i}}{s_{i}}\right)^{m_{i}}} \mathrm{~d} s \\
= & 1-\int_{0}^{\infty} \frac{\Gamma\left(m_{i}, m_{i}\left(\frac{\gamma_{\mathrm{th}}}{s}\right)^{v_{i}}\right)}{\Gamma\left(m_{i}\right)} \frac{\xi}{\sqrt{2 \pi} \sigma_{i} s} \\
& \times \exp \left[-\frac{\left(10 \log _{10} s-\tilde{\mu}_{i}(\rho, \theta)\right)^{2}}{2 \sigma_{i}^{2}}\right] \mathrm{d} s,
\end{aligned}
$$

where $\Gamma(z, t)=\int_{t}^{\infty} x^{z-1} e^{-x} \mathrm{~d} x$ is the incomplete gamma function.

Moreover, let $x=\left(10 \log _{10} s-\tilde{\mu}_{i}(\rho, \theta)\right) /\left(\sqrt{2} \sigma_{i}\right)$, we can further obtain

$$
\begin{aligned}
\operatorname{Pr}\left(\gamma_{i}<\gamma_{\text {th }}\right)= & 1-\frac{1}{\sqrt{\pi} \Gamma\left(m_{i}\right)} \\
& \cdot \int_{-\infty}^{+\infty} \Gamma\left(m_{i}, m_{i}\left(\frac{\gamma_{\text {th }}}{10^{\frac{\sqrt{2} \sigma_{i} x+\tilde{\mu}_{i}(\rho, \theta)}{10}}}\right)^{v_{i}}\right) \\
& \times \exp \left(-x^{2}\right) \mathrm{d} x .
\end{aligned}
$$

Then, by appalying proposition 2, Equation (24) can be further derived as

$$
\operatorname{Pr}\left(\gamma_{i}<\gamma_{\text {th }}\right) \cong 1-\frac{\sum_{n=1}^{N_{p}} H_{n} \Gamma\left(m_{i}, m_{i}\left(\frac{\gamma_{\text {th }}}{10 \frac{\sqrt{2} \sigma_{i} t_{n}+\tilde{\mu}_{i}(\rho, \theta)}{10}}\right)^{v_{i}}\right)}{\sqrt{\pi} \Gamma\left(m_{i}\right)}
$$

which completes the proof.

\section{Abbreviations}

CDF, cumulative distribution function; DAS, distributed antenna system; MS, mobile station; PDF, probability density function; SNR, signal-to-noise ratio; ST, selective transmission.

\section{Competing interests}

The authors declare that they have no competing interests.

\section{Author details}

${ }^{1}$ Key Laboratory for Information System Security of Ministry of Education, School of Software, Tsinghua University, Beijing 100084, China. ${ }^{2}$ National Mobile Communications Research Laboratory, Southeast University, Nanjing 210096, China

Received: 21 January 2013 Accepted: 21 May 2013

Published: 31 May 2013

\section{References}

1. JY Wang, JB Wang, XY Dang, M Lin, Y Jiao, M Chen, in 12th IEEE International Conference on Communication and Technology, System capacity analysis of downlink distributed antenna systems over composite channels, Nanjing, 11-14 November 2010, pp. 1076-1079

2. XH You, DM Wang, B Sheng, XQ Gao, XS Zhao, M Chen, Cooperative distributed antenna systems for mobile communications. IEEE Wireless Commun. 17(3), 35-43 (2010) 
3. JY Wang, JB Wang, M Chen, System capacity analysis and antenna placement optimization for downlink transmission in distributed antenna systems. Wireless Personal Commun. (2012). doi:10.1007/s11277-012-0827-8

4. W Roh, A Paulraj, in In Proceedings of the IEEE Vehicular Technology Conference, vol. 2. MIMO channel capacity for the distributed antenna systems (IEEE, Piscataway, 2002), pp. 706-709

5. H Zhu, Performance comparison between distributed antenna and microcellular systems. IEEE J. Selected Areas in Commun. 29(6), 1151-1163 (2011)

6. V Nikolopoulos, M Fiacco, S Stavrou, SR Saunders, Narrowband fading analysis of indoor distributed antenna systems. IEEE Antennas Wireless Propagation Lett. 2(1), 89-92 (2003)

7. MK Simon, MS Alouini, Digital Communication over Fading Channels, (2nd edn.) (Wiley, New York, 2005)

8. L Dai, S Zhou, Y Yao, Capacity analysis in CDMA distributed antenna systems. IEEE Trans. Wireless Commun. 4(6), 2613-2620 (2005)

9. M Matthaiou, N Chatzidiamantis, G Karagiannidis, A new lower bound on the Ergodic capacity of distributed MIMO systems. IEEE Signal Process. Lett. 18(4), 227-230 (2011)

10. HM Chen, JB Wang, M Chen, Outage capacity study of the distributed MIMO system with antenna cooperation. Wireless Pers. Commun. 59(4), 599-605 (2011)

11. J Zhang, JG Andrews, in IEEE Trans. on Wireless Commun. vol. 7. Distributed antenna systems with randomness. (IEEE, Piscataway, 2008), pp. 3636-3646

12. HM HM Chen, JB Wang, M Chen, Outage performance of distributed antenna systems over shadowed Nakagami-m fading channels. Euro. Trans. Telecommun. 20(5), 531-535 (2009)

13. CHM de Lima, M Bennis, M Latva-aho, in IEEE Transactions on Vehicular Technology Statistical analysis of self-organizing heterogeneous networks with biased cell association and interference avoidance, (IEEE, Piscataway, 2013, in press)

14. EW Stacy, A generalization of the gamma distribution. Ann. Math. Stat. 33(3), 1187-1192 (1962)

15. Y Shen, Y Tang, T Kong, S Shao, Optimal antenna location for STBC-OFDM downlink with distributed transmit antennas in linear cells. IEEE Commun. Lett. 11(5), 387-389 (2007)

16. LL Yang, W Fang, Performance of distributed-antenna DS-CDMA systems over composite lognormal shadowing and Nakagami-m-fading channels. IEEE Trans. Vehicular Technol. 58(6), 2872-2883 (2009)

17. J Park, E Song, W Sung, Capacity analysis for distributed antenna systmes using cooperative transmission schemes in fading channels. IEEE Trans. Wireless Commun. 8(2), 586-592 (2009)

18. W Feng, Y Li, S Zhou, J Wang, M Xia, in IEEE Wireless Communications and Networking Conference, Downlink capacity of distributed antenna systems in a multi-cell environment, Budapest, 5-8 April 2009, pp. 1-5

19. A Goldsmith, Wireless Communication (Cambridge University Press, New York, 2005)

20. R Burden, J Faires, Numerical Analysis Fourth Edition (PWS KENT Publishing Company, Boston, 1989)

21. IS Gradshteyn, IM Ryzhik, Table of Integrals, Series, and Products (7th edn.) (Academic Press, New York, 2007)

22. M Abramowitz, I Stegun, Handbook of Mathematical Functions with Formulas, Graphs, and Mathematical Tables (9th edn.) (Dover Publications, New York, 1970)

doi:10.1186/1687-1499-2013-147

Cite this article as: Cai et al.: Probabilistic analysis of system outage in distributed antenna systems with composite channels. EURASIP Journal on Wireless Communications and Networking 2013 2013:147.

\section{Submit your manuscript to a SpringerOpen ${ }^{\circ}$ journal and benefit from:}

- Convenient online submission

- Rigorous peer review

- Immediate publication on acceptance

- Open access: articles freely available online

- High visibility within the field

- Retaining the copyright to your article

Submit your next manuscript at $\boldsymbol{\wedge}$ springeropen.com 